\title{
THE MAXIMAL DIMENSION OF UNITAL SUBALGEBRAS OF THE MATRIX ALGEBRA
}

\author{
A. L. AGORE
}

\begin{abstract}
We prove that over a field of characteristic zero the maximal dimension of a proper unital subalgebra in the $n \times n$ matrix algebra is $n^{2}-n+1$ and furthermore this upper bound is attained for the so-called parabolic subalgebras. We also investigate the corresponding notion of parabolic coideals for matrix coalgebras and prove that the minimal dimension of a non-zero coideal of the matrix coalgebra $\mathcal{M}^{n}(k)$ is $n-1$.
\end{abstract}

\section{INTRODUCTION}

The problem of finding the minimal/maximal dimension of proper subobjects (possibly satisfying some extra assumptions) of a given mathematical object is a natural one and it was studied in various settings. The first result in this direction was proved by Schur [13] and asserts that $\left[\frac{n^{2}}{4}\right]+1$ is an upper bound for the dimensions of commutative subalgebras of $\mathcal{M}_{n}(\mathbb{C})$. Later on, Jacobson [6] showed that the same bound is valid over any field $K$. An elegant and simpler proof of Schur's theorem was given by Mirzakhani in [10]. These results allowed for the introduction of the Schur invariant in Lie algebra theory as the maximal dimension of abelian subalgebras of a given Lie algebra. A vast literature emerged from the study of this invariant which plays an important role in many aspects of Lie algebra theory, see for example $[3,11]$ and the references therein. To give just one example, the Schur invariant has been completely determined for semisimple Lie algebras by Malcev in [8]. In this note we address the problem of finding the maximal dimension of subalgebras of the matrix algebra $\mathcal{M}_{n}(K)$ over a field $K$ of characteristic zero. Our main result is Theorem 2.4 which gives an upper bound for the dimension of subalgebras in $\mathcal{M}_{n}(K)$ and it proves that this maximal dimension is attained for the so-called parabolic subalgebras [16], which are the associative algebra counterpart of the well-known parabolic Lie subalgebras (see $[17,18]$ for more details). A subalgebra $\mathcal{A}$ of $\mathcal{M}_{n}(K)$ is called parabolic if it is similar to an algebra consisting of all matrices having non-overlapping blocks of $n_{i} \times n_{i}$ matrices on the diagonal, $i=1,2, \ldots, s$, with non-zero entries only in these blocks or above them, where $\left(n_{1}, n_{2}, \ldots, n_{s}\right)$ is a partition of $n$. As a consequence of Theorem 2.4 we prove in Corollary 2.6 that the maximal dimension

2010 Mathematics Subject Classification. 16D70, $16 \mathrm{Z} 05$.

Key words and phrases. matrix (co)algebra, parabolic subalgebras of matrix algebras, parabolic coideals of matrix coalgebras, maximal dimension of subalgebras.

The author is Postdoctoral Fellow of the Fund for Scientific Research-Flanders (Belgium) (F.W.O. Vlaanderen). This research is part of the grant no. 88/05.10.2011 of the Romanian National Authority for Scientific Research, CNCS-UEFISCDI. 
of a proper unital subalgebra of $\mathcal{M}_{n}(K)$ is equal to $n^{2}-n+1$. A related result was obtained in [1] where it was proved that $\mathcal{M}_{n}(K)$ has no subalgebras of codimension equal to 1 . A somewhat similar problem was considered recently in $[3,4]$ in the context of Lie algebras and in [9] for Lie algebras as well as for associative algebras. In the above mentioned papers the authors are interested in finding bounds for the dimensions of abelian subalgebras or abelian ideals.

The paper ends with a brief discussion on matrix coalgebras (see Section 1 for the definition and further details). We introduce the notion of parabolic coideals for matrix coalgebras and derive some of their features based on the duality between the matrix algebra and the matrix coalgebra. As an application, we prove that the minimal dimension of a non-zero coideal of the matrix coalgebra $\mathcal{M}^{n}(K)$ is $n-1$.

\section{Preliminaries}

Unless otherwise stated, all vector spaces, linear maps, algebras and coalgebras are over an arbitrary field $K$. In some specific cases we will assume that $K$ is algebraically closed of characteristic zero. For a vector space $V$ we denote by $V^{*}:=\operatorname{Hom}_{K}(V, K)$ the dual of $V$. If $S$ is a subset of $V$ we set $S^{\perp}:=\left\{v \in V^{*} \mid v(S)=0\right\}$. Similarly, if $Y$ is a subset of $V^{*}$ we denote $Y^{\perp}:=\{x \in V \mid f(x)=0$, for all $f \in Y\}$. If $X$ is a subspace of the vector space $\mathrm{V}$ then the codimension of $X$ is $\operatorname{dim}_{K}(V / X)$.

By an algebra (coalgebra) we mean an associative (coassociative), unital (counital) algebra (coalgebra) over $K$. If $A$ is an algebra, $\operatorname{rad} A$ stands for the Wedderburn-Artin radical of $A$. Wedderburn's main theorem ([7, Theorem 2.17]) is a fundamental result on the structure of finite dimensional algebras, being the associative algebra counterpart of the famous Levi decomposition of a Lie algebra: it asserts that for any finite dimensional algebra $A$ over a field $K$ of characteristic zero, there exists a semisimple subalgebra $S$ of $A$ isomorphic to $A / \operatorname{rad} A$ such that $A=S \oplus \operatorname{rad} A$. Moreover, over an algebraically closed field any semisimple finite-dimensional algebra is a direct sum of matrix algebras over the same field. We refer the reader to [7, Chapter II] for a detailed account on the Wedderburn-Artin theory. For an arbitrary integer $n \geq 2$ we let $\mathcal{M}_{n}(K)$ denote the algebra of $n \times n$ matrices over the field $K$ while $\mathcal{U}_{n}(K)$ and $\overline{\mathcal{U}_{n}(K)}$ stand for the set of all upper triangular matrices in $\mathcal{M}_{n}(K)$, respectively the set of all strictly upper triangular matrices in $\mathcal{M}_{n}(K)$. We denote by $e_{i, j} \in \mathcal{M}_{n}(K)$ the matrix having 1 in the $(i, j)^{t h}$ position and zeros elsewhere. If $\mathcal{A}$ is a subset of $\mathcal{M}_{n}(K)$, then we denote $C \mathcal{A} C^{-1}=\left\{C A C^{-1} \mid A \in \mathcal{A}\right\}$.

If $A$ is a finite dimensional algebra then $A^{*}$ has a natural coalgebra structure called the dual coalgebra on $A$. In the same manner, if $C$ is a (not necessarily finite dimensional) coalgebra then $C^{*}$ inherits an algebra structure called the dual algebra on $C \cdot \mathcal{M}^{n}(K)$ denotes the matrix coalgebra, i.e. $\mathcal{M}^{n}(K)=\mathcal{M}_{n}(K)$ as vector spaces with the coalgebra structure given for all $i, j=1,2, \ldots, n$ by:

$$
\begin{aligned}
& \Delta: \mathcal{M}^{n}(K) \rightarrow \mathcal{M}^{n}(K) \otimes \mathcal{M}^{n}(K), \quad \Delta\left(e_{i, j}\right)=\sum_{k=1}^{n} e_{i, k} \otimes e_{k, j} \\
& \varepsilon: \mathcal{M}^{n}(K) \rightarrow K, \quad \varepsilon\left(e_{i, j}\right)=\delta_{i, j}
\end{aligned}
$$


It is well-known that $\mathcal{M}_{n}(K)^{*}$ and $\mathcal{M}^{n}(K)$ are isomorphic as coalgebras. Similarly, $\mathcal{M}^{n}(K)^{*}$ and $\mathcal{M}_{n}(K)$ are isomorphic as algebras. For all unexplained notions or results from the theory of coalgebras we refer the reader to $[2,15]$.

We also make use of a well-known result on the dimension of subspaces of nilpotent matrices which first appeared in [5]. An improved version of this result appears in [14], where the cardinality assumption on the field $K$ is removed. More precisely, the result in [14] states that if $\mathcal{S}$ is a subspace of the vector space of $n \times n$ matrices over an arbitrary field $K$ and $\mathcal{S}$ consists of nilpotent matrices, then the maximal dimension of $\mathcal{S}$ is $\frac{n(n-1)}{2}$. Moreover, if equality holds then $\mathcal{S}$ is conjugate to $\overline{\mathcal{U}_{n}(K)}$.

\section{Parabolic SUbalgebras of Matrix Algebras}

By analogy with Lie algebra theory ([17]), a proper subalgebra $\mathcal{A}$ of the matrix algebra $\mathcal{M}_{n}(K)$ is called parabolic [16] if it is similar to an algebra which contains $\mathcal{U}_{n}(K)$, i.e. there exists an invertible matrix $U$ such that $\mathcal{U}_{n}(K) \subseteq U \mathcal{A} U^{-1}$. As we will see, this concept will play an important role in determining the maximal dimension of a subalgebra in a matrix algebra. It was proved in [16] that $\mathcal{A}$ is a parabolic subalgebra of $\mathcal{M}_{n}(K)$ if and only if there exists a set of positive integers $n_{1}, n_{2}, \ldots, n_{s}$ with $\sum_{i=1}^{s} n_{i}=n$ such that $\mathcal{A}$ is similar to the algebra of all matrices having non-overlapping blocks of $n_{i} \times n_{i}$ matrices on the diagonal with non-zero entries only in these blocks or above them. Moreover, it is straightforward to see that $\operatorname{dim} A=\frac{n^{2}}{2}+\sum_{i=1}^{s} \frac{n_{i}^{2}}{2}$.

Definition 2.1. A parabolic subalgebra $\mathcal{A}$ of $\mathcal{M}_{n}(K)$ determined by the set of positive integers $n_{1}, n_{2}, \ldots, n_{s}$ will be called a parabolic subalgebra of type $\left(n_{1}, n_{2}, \ldots, n_{s}\right)$. If $s=2$ then $\mathcal{A}$ will be called a maximal parabolic subalgebra.

Our next result shows that a maximal parabolic subalgebra is in fact a maximal proper subalgebra of $\mathcal{M}_{n}(K)$.

Proposition 2.2. The maximal parabolic subalgebras are maximal proper subalgebras of $\mathcal{M}_{n}(K)$.

Proof. Consider $\mathcal{A}$ to be a parabolic subalgebra having on the diagonal two blocks $U$ and $V$ of dimensions $l \times l$ and respectively $(n-l) \times(n-l)$ with $l \geq 1$ and assume there exists a subalgebra $\mathcal{T}$ of $\mathcal{M}_{n}(K)$ such that $\mathcal{A} \subset \mathcal{T}, \mathcal{A} \neq \mathcal{T}$. The elements of $\mathcal{T}$ are linear combinations of the matrix units $e_{i, j}$ and there must be an element $x \in \mathcal{T}-\mathcal{A}$ that has a non-zero entry $\alpha$ in a position $(i, j)$ with $i>l$ and $j \leqslant l$. Then $e_{i, j} \in \mathcal{T}$ since $e_{i, j}=\alpha^{-1} e_{i, i} x e_{j, j}$. Since $i>l, e_{m, i} \in \mathcal{A}$ for all $m$ and hence $e_{m, j}=e_{m, i} e_{i, j}$ belongs to $\mathcal{T}$ for all $m$. Similarly $e_{j, k} \in \mathcal{A}$ for all $k$ and so $e_{i, k}=e_{i, j} e_{j, k} \in \mathcal{T}$ for all $k$. Thus if $e_{i, j}$ belongs to $\mathcal{T}$, then so do all the matrix units from row $i$ and column $j$. Using the same reasoning for all these elements we can conclude that $\mathcal{T}=\mathcal{M}_{n}(K)$ which finishes the proof. 
Example 2.3. The parabolic subalgebras of $\mathcal{M}_{3}(K)$ are similar to the following subalgebras:

$$
\left(\begin{array}{ccc}
K & K & K \\
0 & K & K \\
0 & 0 & K
\end{array}\right) \quad\left(\begin{array}{ccc}
K & K & K \\
K & K & K \\
0 & 0 & K
\end{array}\right) \quad\left(\begin{array}{ccc}
K & K & K \\
0 & K & K \\
0 & K & K
\end{array}\right)
$$

The last two algebras are maximal proper subalgebras of $\mathcal{M}_{3}(K)$.

If $K$ is an algebraically closed field of characteristic zero and $\mathcal{A}$ is a finite dimensional $K$-algebra then by Wedderburn's theorem [7], $\mathcal{A}$ has a semisimple part which is a direct sum of matrix algebras over $K$ of dimensions $n_{1}^{2}, n_{2}^{2}, \ldots, n_{s}^{2}$. In order for $\mathcal{A}$ to be a subalgebra of $\mathcal{M}_{n}(K)$ we need to have $\sum_{i=1}^{s} n_{i} \leq n$. However, since we are trying to maximize the dimension of $\mathcal{A}$ we will assume that $\sum_{i=1}^{s} n_{i}=n$.

Theorem 2.4. Let $K$ be an algebraically closed field of characteristic zero and $\mathcal{A}$ a subalgebra of $\mathcal{M}_{n}(K)$ whose semisimple part $S$ is a direct sum of matrix algebras over $K$ of dimensions $n_{1}^{2}, n_{2}^{2}, \ldots, n_{s}^{2}$ with $\sum_{i=1}^{s} n_{i}=n$. Then $\operatorname{dim} \mathcal{A} \leq \frac{n^{2}}{2}+\sum_{i=1}^{s} \frac{n_{i}^{2}}{2}$. Moreover, if equality holds then $A$ is a parabolic subalgebra of $\mathcal{M}_{n}(K)$.

Proof. By Wedderburn's main theorem [7, Theorem 2.17] we have $\mathcal{A}=S \oplus \operatorname{rad} \mathcal{A}$. Let $\operatorname{dim} \mathcal{A}=d$ and $\operatorname{dim} \operatorname{rad} \mathcal{A}=r$. It follows that $\sum_{i=1}^{s} n_{i}^{2}+r=d$. Remark that each $n_{i} \times n_{i}$ matrix subalgebra of $S$ contains its nilpotent subalgebra of strictly upper triangular matrices. This implies that $\mathcal{A}$ contains, all together, a subalgebra of nilpotent matrices of dimension $\sum_{i=1}^{s} \frac{n_{i}\left(n_{i}-1\right)}{2}+r$. Using Gerstenhaber's result ([5]) on subspaces of nilpotent matrices we must have $\sum_{i=1}^{s} \frac{n_{i}\left(n_{i}-1\right)}{2}+r \leq \frac{n(n-1)}{2}$. Since $\sum_{i=1}^{s} n_{i}^{2}+r=d$ we get $d \leq \frac{n(n-1)}{2}+\sum_{i=1}^{s} \frac{n_{i}\left(n_{i}+1\right)}{2}$. The conclusion now follows by using $\sum_{i=1}^{s} n_{i}=n$.

Suppose now that equality holds. It follows that $r=\frac{n^{2}}{2}-\sum_{i=1}^{s} \frac{n_{i}^{2}}{2}$ and so $\mathcal{A}$ contains a subalgebra of nilpotent matrices of dimension $\frac{n(n-1)}{\frac{2}{U_{n}(K)}}$. Gerstenhaber's result implies that any such subspace of $\mathcal{M}_{n}(K)$ is conjugate to $\overline{\mathcal{U}_{n}(K)}$. Since for any invertible matrix $C \in \mathcal{M}_{n}(K)$ the map $u: \mathcal{A} \rightarrow C \mathcal{A} C^{-1}$ which takes any $A \in \mathcal{A}$ to $C A C^{-1}$ is an algebra isomorphism we may assume without loss of generality that $\mathcal{A}$ contains $\overline{\mathcal{U}_{n}(K)}$. However, by looking at dimensions one sees that $\overline{\mathcal{U}_{n}(K)}$ is not all of $\mathcal{A}$. Now since the semisimple part of $\mathcal{A}$ is a direct sum of matrix algebras of dimensions $n_{1}, n_{2}, \ldots, n_{s}$ with $\sum_{i=1}^{s} n_{i}=n$ it follows that each $e_{i i} \in \mathcal{A}$ for all $i=1,2, \ldots, n$. Therefore, $\mathcal{A}$ contains $\mathcal{U}_{n}(K)$ and the proof is now finished.

Proposition 2.5. Let $K$ be an algebraically closed field of characteristic zero. Then the proper subalgebras of maximum dimension in $\mathcal{M}_{n}(K)$ are the parabolic subalgebras of type $(1, n-1)$ and respectively $(n-1,1)$.

Proof. By Theorem 2.4 we know that the upper bound for the dimension of a subalgebra in $\mathcal{M}_{n}(K)$ is attained when the subalgebra is parabolic. In what follows we will prove that this bound is maximal precisely when the subalgebra is parabolic of type $(1, n-1)$ and respectively $(n-1,1)$. Since the dimension of a parabolic subalgebra of $\mathcal{M}_{n}(K)$ is $\frac{n^{2}}{2}+\sum_{i=1}^{s} \frac{n_{i}^{2}}{2}$ in order to maximize its dimension we need to have $\sum_{i=1}^{s} n_{i}^{2}$ as large 
as possible. We denote by $S:=n_{1}^{2}+n_{2}^{2}+\ldots+n_{i}^{2}+\left(n-n_{1}-n_{2}-\ldots-n_{i}\right)^{2}$ and by $S^{\prime}:=n_{1}^{2}+n_{2}^{2}+\ldots+n_{i-1}^{2}+\left(n-n_{1}-n_{2}-\ldots-n_{i-1}\right)^{2}$, where $n_{t}$ are positive integers for all $t=1,2, \ldots, i$, such that $n_{1}+n_{2}+\ldots+n_{i}<n$. The proof will be finished once we show that $S<S^{\prime}$. Indeed, this follows by noticing that $S=S^{\prime}-2 n_{i}\left(n-n_{1}-n_{2}-\ldots-n_{i}\right)<S^{\prime}$. Therefore we need to consider parabolic subalgebras of type $(l, n-l)$, with $l \geq 1$. Then $\sum_{i=1}^{s} n_{i}^{2}=l^{2}+(n-l)^{2}$. Now it can easily be seen that for $1<l<n-1$ we have $l^{2}+(n-l)^{2}<1+(n-1)^{2}$ and the conclusion follows.

Corollary 2.6. Let $K$ be a field of characteristic zero. Then the maximal dimension of a proper subalgebra of the matrix algebra $\mathcal{M}_{n}(K)$ is $n^{2}-n+1$.

Proof. If $K$ is an algebraically closed field of characteristic zero then the assertion follows from Proposition 2.5. We will prove that the algebraically closed assumption on the field $K$ can be dropped by simply extending the coefficients to the algebraic closure $\bar{K}$ of $K$. Indeed, let $A$ be a $K$-subalgebra of $\mathcal{M}_{n}(K)$. We have:

$$
\bar{K} \simeq \bar{K} \otimes_{K} K \subset \bar{K} \otimes_{K} A \subseteq \bar{K} \otimes_{K} \mathcal{M}_{n}(K) \simeq \mathcal{M}_{n}(\bar{K})
$$

where the last isomorphism follows from [12, Lemma 7.130]. Therefore, $\bar{K} \otimes_{K} A$ is a $\bar{K}$ subalgebra of $\mathcal{M}_{n}(\bar{K})$ and since $\operatorname{dim}_{K}(A)=\operatorname{dim}_{\bar{K}}\left(\bar{K} \otimes_{K} A\right)$ the conclusion follows.

We end the paper by looking at the matrix coalgebra case. It is well-known that $X \subseteq$ $\mathcal{M}^{n}(K)$ is a coideal if and only if $X^{\perp}$ is a subalgebra of $\mathcal{M}_{n}(K)$ (see [15, Proposition 1.4.6] for a more general statement). In light of this bijection we introduce the following:

Definition 2.7. A coideal $X \subseteq \mathcal{M}^{n}(K)$ will be called parabolic if $X^{\perp}$ is a parabolic subalgebra of $\mathcal{M}_{n}(K)$. If $X^{\perp}$ is a parabolic subalgebra of type $\left(n_{1}, n_{2}, \ldots, n_{s}\right)$ then $X$ will be called a parabolic coideal of type $\left(n_{1}, n_{2}, \ldots, n_{s}\right)$ as well. If $s=2$ then $X$ will be called a minimal parabolic coideal.

The parabolic coideals of $\mathcal{M}^{n}(K)$ can be characterized as follows:

Proposition 2.8. Let $X$ be a parabolic coideal of type $\left(n_{1}, n_{2}, \ldots, n_{s}\right)$ of the matrix coalgebra $\mathcal{M}^{n}(K)$. Then $X$ is the coideal of all matrices having non-overlapping blocks of $n_{i} \times n_{i}$ matrices on the diagonal with non-zero entries only below these blocks.

Proof. If $\mathcal{A}$ is a subalgebra of $\mathcal{M}^{n}(K)^{*} \simeq \mathcal{M}_{n}(K)$ (isomorphism of algebras) then $\mathcal{A}^{\perp}$ is a coideal in $\mathcal{M}^{n}(K)$. The conclusion follows by a straightforward computation.

Proposition 2.9. The minimal parabolic coideals are minimal proper coideals of $\mathcal{M}^{n}(K)$.

Proof. Let $X$ be a minimal parabolic coideal of type $(l, n-l)$ and assume there exists a coideal $Y$ of $\mathcal{M}^{n}(K)$ such that $Y \subset X, Y \neq X$. Then $X^{\perp} \subset Y^{\perp}$ and $X^{\perp}$ is a maximal parabolic subalgebra of $\mathcal{M}_{n}(K)$. Using Proposition 2.2 we obtain $Y^{\perp}=\mathcal{M}_{n}(K)$. This implies $Y=\{0\}$ and the proof is finished.

Proposition 2.10. Let $K$ be an algebraically closed field of characteristic zero. Then the non-zero coideals of minimal dimension in $\mathcal{M}^{n}(K)$ are those parabolic coideals $X$ for which $X^{\perp}$ is a parabolic subalgebra of type $(1, n-1)$ and respectively $(n-1,1)$. 
Proof. For any finite dimensional vector space $V$, and any subspace $X$ of $V^{*}$ we have $\operatorname{dim}_{K} X^{\perp}=\operatorname{dim}_{K} V^{*} / X$. Therefore, for any coideal $X$ in $\mathcal{M}^{n}(K)$ we have $\operatorname{dim}_{K} X^{\perp}=$ $\operatorname{dim}_{K} \mathcal{M}^{n}(K) / X$. Collaborating this result with the bijection between the coideals of $\mathcal{M}^{n}(K)$ and the subalgebras of $\mathcal{M}_{n}(K)$ ([15]), it follows that a coideal $X$ in $\mathcal{M}^{n}(K)$ has maximal codimension precisely when the subalgebra $X^{\perp}$ of $\mathcal{M}_{n}(K)$ has maximal dimension. The conclusion now follows by Proposition 2.5.

Corollary 2.11. Let $K$ be a field of characteristic zero. Then the minimal dimension of a non-zero coideal in $\mathcal{M}^{n}(K)$ is $n-1$.

\section{REFERENCES}

[1] Agore, A.L., Militaru, G. - Extending structures, Galois groups and supersolvable associative algebras, 30 pages, to appear in Monatsh. für Mathematik, DOI:10.1007/s00605-015-0814-8.

[2] Brzeziński, T., Wisbauer, R. - Corings and comodules, London Math. Soc. Lect. Note Ser. 309, Cambridge University Press, Cambridge, 2003.

[3] Burde, D., Ceballos, M. - Abelian ideals of maximal dimension for solvable Lie algebras, J. Lie Theory 22 (2012), 741-756.

[4] Ceballos, M., Towers, D.A. - On abelian subalgebras and ideals of maximal dimension in supersolvable Lie algebras, J. Pure Appl. Algebra 218 (2014), 497-503.

[5] Gerstenhaber, M. - On nilalgebras and linear varieties of nilpotent matrices I, Amer. J. Math. 80 (1958), 614-622.

[6] Jacobson, N. - Schur's theorems on commutative matrices, Bull. AMS 50 (1944), 431-436.

[7] Knapp, A.W. - Advanced algebra, Birkhauser Boston, Inc., Boston, MA (2007).

[8] Malcev, A. - Commutative subalgebras of semi-simple Lie algebras, Amer. Math. Soc. Transl. 40 (1951), p. 214.

[9] Milentyeva, M. V. - On the dimensions of commutative subalgebras and subgroups, J. Math. Sciences 149 (2008), 1135-1145.

[10] Mirzakhani, M. - A simple proof of a theorem of Schur, Amer. Math. Monthly 105 (1998), 260-262.

[11] Nesterenko, M., Popovych, R. - Contractions of low-dimensional Lie algebras, J. Math. Phys. 47 (2006), 123515, $45 \mathrm{pp}$.

[12] Rotman, J. J. - Advanced modern algebra, Second edition, Graduate Studies in Mathematics, 114 (2010).

[13] Schur, I. - Zur Theorie vertauschbarer Matrizen, J. Reine Angew. Math. 130 (1905), 66-76.

[14] Serezhkin, V.N. - Linear transformations preserving nilpotency, Vestsi Akad. Navuk BSSR Ser. Fiz.-Mat. Navuk 5 (1985), 46-50.

[15] Sweedler, M.E. - Hopf Algebras, Benjamin New York, 1969

[16] Wang, D., Pan, H., Wang, X. - Non-linear maps preserving ideals on a parabolic subalgebra of a simple algebra, Czechoslovak Math. J. 60 (2010), 371-379.

[17] Wang, D., Yu, Q. - Derivations of the parabolic subalgebras of the general linear Lie algebra over a commutative ring, Linear Algebra Appl. 418 (2006), 763-774.

[18] Warner, G. - Harmonic Analysis on Semi-Simple Lie Groups I, Springer, Berlin, 1972.

Faculty of Engineering, Vrije Universiteit Brussel, Pleinlaan 2, B-1050 Brussels, Belgium and "Simion Stoilow" Institute of Mathematics of the Romanian Academy, P.O. Box 1-764, 014700 Bucharest, Romania

E-mail address: ana.agore@vub.ac.be and ana.agore@gmail.com 